\title{
VARIABLES COGNITIVAS Y METACOGNITIVAS EN LA RESOLUCIÓN DE PROBLEMAS DE QUÍMICA: PROPUESTA DE ESTRATEGIAS DIDÁCTICAS
}

\author{
Joan Josep Solaz-Portolés* \\ IES Benaguasil/ C.A. Valencia de la UNED, España
}

Recebido em 20/9/09; aceito em 3/2/10; publicado na web em 21/5/10

\begin{abstract}
COGNITIVE AND METACOGNITIVE VARIABLES IN CHEMISTRY PROBLEM SOLVING: A PROPOSAL OF DIDACTIC STRATEGIES. This paper presents and classifies the cognitive and metacognitive variables involved in the processes that students execute in problem solving. Moreover, it shows how these variables affect the students success in problem solving. These variables are classified in: piagetian and neo-piagetian, representational, metacognitive and transfer of learning. In the first group of variables it is discussed formal reasoning ability and other neo-piagetian factors. In the second group of variables it is analysed mental models and external representations. Implications for chemistry education are collected as a proposal of didactic strategies in the classroom.
\end{abstract}

Keywords: problems solving; cognitive and metacognitive variables; didactic strategies.

\section{INTRODUCCIÓN}

La resolución de problemas desempeña un papel clave en la educación científica. Es una actividad muy importante en los centros educativos porque se utiliza para entrenar a nuestros estudiantes a aplicar la metodología científica. En ella, el estudiante externaliza el proceso constructivo de aprender, convierte en acciones los conceptos, las proposiciones o los ejemplos, a través, fundamentalmente, de las interacciones con el profesor y los materiales instruccionales. ${ }^{1}$ También puede ser utilizada la resolución de problemas como actividad motivadora en la clase de química. Sin embargo, nuestros alumnos no suelen tener éxito en la aplicación de los conocimientos adquiridos a la resolución de problemas, ya sea en el contexto escolar o en la vida cotidiana. Esta circunstancia parece ser especialmente destacada cuando se trata de disciplinas científicas. ${ }^{2,3}$

Las últimas cuatro décadas se han llevado a cabo gran cantidad de estudios sobre resolución de problemas, y de hecho, existe un consenso creciente en relación a los procesos mentales y factores cognitivos implicados en la resolución de problemas. ${ }^{4}$ La literatura nos sugiere que el éxito en la resolución de problemas depende de la combinación de un intenso conocimiento de la materia, de las estrategias de resolución de problemas, y de componentes actitudinales. ${ }^{5}$

En el presente trabajo, a partir del análisis de los conocimientos y procesos necesarios para resolver problemas en el aula de química, vamos a plantear estrategias didácticas que puedan mejorar significativamente el rendimiento de nuestros estudiantes en la resolución de problemas. Para ello, haremos uso de recientes investigaciones y revisiones bibliográficas que se han centrado en estas cuestiones. Las variables que inciden en la resolución de problemas las vamos a clasificar en: piagetianas y neopiagetianas, representacionales, metacognitivas y de transferencia de aprendizajes.

\section{VARIABLES PIAGETIANAS Y NEOPIAGETIANAS}

Solaz-Portolés y Sanjosé ${ }^{6}$ nos presentan una visión global de las investigaciones que se han efectuado sobre estas variables cuando están implicadas en la resolución de problemas. Las variables discutidas son: habilidad de razonamiento formal, M-espacio, factor campo (dependiencia/independencia de campo), dimensión cognitiva

\footnotetext{
*e-mail: jjsolaz@valencia.uned.es
}

móvil/fija, capacidad de la memoria de trabajo y colaboración entre estudiantes en las tareas de resolución de problemas. Veamos qué son cada una de ellas y su influencia en la resolución de problemas.

La capacidad de razonamiento formal constituye la última etapa en la construcción del conocimiento en la teoría de Piaget. En ella, se consigue utilizar y representar conceptos abstractos y generar estructuras de conceptos científicos. De acuerdo con los seguidores de Piaget, los alumnos que no hayan accedido a la etapa de las operaciones formales no tienen posibilidad de comprender y asimilar conceptos y principios científicos y, en consecuencia, son incapaces de resolver problemas.

El M-espacio o capacidad mental es una variable que explica el incremento de la capacidad de procesamiento de la información con la edad. El factor campo representa la habilidad del sujeto para extraer información en una amplia variedad de contextos instruccionales; así, a los aprendices que tiene mayor dificultad que otros para separar la señal del ruido se les clasifica como dependientes de campo. La dimensión cognitiva móvil/fija resulta de una combinación de las dos anteriores variables.

La memoria de trabajo tiene la función de almacenar información temporalmente. Los investigadores usan el concepto de capacidad de la memoria de trabajo para representar la cantidad de información activada y retenida mientras se ejecutan tareas cognitivas. Dicha capacidad es limitada y, la imposición de un exceso de demanda de procesamiento o de almacenamiento de información, conduce inexorablemente a pérdidas de información. ${ }^{7}$

La teoría de Piaget hace explícita referencia a la importancia del contexto social en el aprendizaje. Diversos seguidores de Piaget han puesto de relieve este aspecto en la resolución de problemas. Defienden la resolución de problemas en el aula de forma cooperativa.

Las investigaciones desarrolladas sobre estas variables han concluido que: existe una relación de proporcionalidad directa entre la capacidad de razonamiento formal y éxito en la resolución de problemas, los alumnos con mayor capacidad mental (M-espacio) e independientes de campo realizan mejor los problemas, los estudiantes de dimensión cognitiva móvil resuelven mejor los problemas creativos y los de dimensión cognitiva fija los que requieren mayor razonamiento formal, la capacidad de la memoria de trabajo es decisiva en la resolución de problemas (mayor capacidad implica mayor facilidad para resolver problemas), y la colaboración entre alumnos en la resolución de problemas en el aula mejora sus habilidades en esta tarea. 


\section{VARIABLES REPRESENTACIONALES}

En otro trabajo de Solaz-Portolés y Sanjosé ${ }^{8}$ se muestran los aspectos más relevantes que los investigadores en la didáctica de las ciencias han ofrecido a la comunidad científica sobre las interrelaciones representaciones-resolución de problemas. En él se habla de las representaciones internas y externas que construyen los sujetos cuando intentan resolver un problema. A las primeras, se les suele denominar modelos mentales. El modelo mental es el modo en que el sujeto solucionador suele almacenar los componentes del problema en su memoria. La representación externa la utilizan los estudiantes para simbolizar objetos y procesos presentes en el enunciado del problema. Las representaciones externas pueden ser, por ejemplo, diagramas, ecuaciones o gráficas.

Cuando un estudiante intenta resolver un problema suele construir varios modelos mentales que puede procesar simultáneamente. La elaboración de dichos modelos mentales esta condicionada por diversas variables: disposición de los conocimientos adecuados para resolver el problema (base de conocimientos del problema), capacidad adecuada de la memoria de trabajo y dominio suficiente de habilidades metacognitivas que permiten la manipulación de modelos mentales (de éstas hablaremos posteriormente).

La teoría de modelos mentales en la resolución de problemas ha sido puesta a prueba, ${ }^{9}$ confirmándose una relación inversa entre el número de modelos mentales que se han de activar y el porcentaje de estudiantes que lo resuelve correctamente, tal y como predice la teoría. Además, se ha demostrado la influencia que ejercen ciertas variables instruccionales en la formación de los modelos necesarios para resolver los problemas. ${ }^{10}$

En relación a los conocimientos necesarios para construir los modelos mentales apropiados para resolver problemas, Solaz-Portolés y Sanjosé ${ }^{11}$ proporcionan una panorámica general de los mismos. Distinguen siete tipos de conocimientos: declarativo, procedimental, esquemático, estratégico, situacional, metacognitivo y de traducción de problemas. El conocimiento declarativo es el conocimiento conceptual, esto es, aquel que incluye contenidos del dominio específico de que se trate: hechos, definiciones y descripciones. El conocimiento procedimental es el tipo de conocimiento que contiene acciones o manipulaciones válidas dentro de un determinado dominio conceptual. El conocimiento situacional hace referencia al conocimiento de los casos, operaciones, procesos, etc. que pueden darse dentro de una determinada área disciplinar. El conocimiento esquemático resulta de la combinación adecuada de los conocimientos conceptual, procedimental y situacional. El conocimiento estratégico ayuda al estudiante a organizar los procesos efectuados durante la resolución del problema. El conocimiento metacognitivo permite planear y controlar el progreso hacia la resolución del problema. Finalmente, el conocimiento en la traducción de problemas posibilita comprender, analizar, interpretar y definir un problema. Se ha constatado que los expertos en resolución de problemas tienen una gran base de conocimientos que está organizada en estructuras bien elaboradas e integradas, en tanto que los novatos, tienen menos conocimientos y peor organizados.

La habilidad para mantener la información en un estado activo es fundamental para integrar información en los sucesivos pasos que se ejecutan cuando se resuelve un problema, incluyendo la construcción y elaboración de los modelos mentales pertinentes. Múltiples estudios dan apoyo a la significativa dependencia entre el éxito en la resolución de problemas y la capacidad de la memoria de trabajo. De hecho, se ha comprobado que cuando la carga informativa de un problema excede a la capacidad de la memoria de trabajo del estudiante, el rendimiento en la resolución del problema cae estrepitosamente. ${ }^{7}$

Por otra parte, el uso de representaciones externas mediante símbolos u objetos puede facilitar las complejas tareas cognitivas que se desarrollan durante la resolución de problemas. ${ }^{8}$ Esto es debido, fundamentalmente, a la reducción de la carga cognitiva que supone tener a mano dichas ilustraciones. Parece ser beneficioso tanto la presentación junto al enunciado del problema de las oportunas representaciones, como el entrenamiento de los estudiantes en el uso de representaciones mientras se efectúan las tareas de resolución de problemas. Este último caso se justifica en función de la asunción, por parte del estudiante, de su imposibilidad para mantener en la memoria de trabajo toda la información que le requiere el problema. Cuando el estudiante dibuja la representación se descarga de parte de la información pero la mantiene igualmente accesible.

\section{VARIABLES METACOGNITIVAS}

El término metacognición hace referencia al conocimiento personal relativo a los propios procesos cognitivos y a todo lo demás relacionado con ellos. Artz y Armour-Thomas ${ }^{12}$ en un estudio sobre los procesos metacognitivos en la resolución de problemas, acaban concluyendo que las interacciones continuas entre las destrezas cognitivas (base de conocimientos) y las metacognitivas resultan trascendentales en la consecución de la resolución de problemas. De la revisión bibliográfica llevada a cabo por Taconis, FergussonHessler y Broekkamp, ${ }^{13}$ se deduce que suministrar a los aprendices guías y criterios para poder juzgar sus procesos y productos durante la resolución de problemas, con una inmediata retroalimentación, parecen ser los prerrequisitos más importantes para adquirir habilidades adecuadas en la resolución de problemas.

La metodología instruccional presentada por Lorenzo, ${ }^{14} \mathrm{de}-$ nominada heurístico de resolución de problemas, intenta ayudar a comprender a los estudiantes los pasos implicados en la resolución de problemas y proporcionarles un enfoque organizativo para abordar los problemas de un modo sistemático. Este enfoque guía hacia un razonamiento cualitativo del problema, antes que a la realización rápida de cálculos. Usa siempre una estrategia hacia atrás reflexiva de los pasos ejecutados en la resolución. La aplicación de esta metodología didáctica exige, entre otras cosas, la resolución de problemas en un ambiente de cooperación entre estudiantes.

\section{VARIABLES DE TRANSFERENCIA DE APRENDIZAJES}

La transferencia de aprendizajes es frecuentemente definida como la habilidad para aplicar lo que ha sido aprendido en un determinado contexto a un nuevo contexto. Tradicionalmente la transferencia ha sido medida examinando si los estudiantes podían aplicar lo que había sido aprendido en un problema a nuevos problemas isomórficos.

Los estudiantes inexpertos cuando resuelven problemas usan con asiduidad analogías entre problemas como guías de resolución. ${ }^{15}$ Sin embargo, cuando el problema fuente (con el que se aprende) y el problema diana (con el que se mide la transferencia) pertenecen a diferentes disciplinas y son superficialmente desemejantes, se hace más difícil la transferencia. ${ }^{16}$

Los resultados de Catrambone y Holyoak ${ }^{17}$ sacan a la luz que puede conseguirse transferencia entre problemas desemejantes mediante entrenamiento con ejemplos y manipulaciones que promuevan la abstracción del esquema de problema (esto es, en definitiva, promover el conocimiento esquemático de problemas). En el proceso de abstracción de esquemas de resolución de problemas, la eficacia de comparar ejemplos de estructura idéntica o muy similar ha sido probada como un buen método para facilitar la transferencia analógica. ${ }^{18}$ Recientemente se ha mostrado que comparar entre sí problemas propuestos se muestra muy eficiente en el proceso de abstracción y recuperación de esquemas de resolución. ${ }^{19}$

El profesorado asume con mucha frecuencia que las relaciones analógicas entre los problemas resueltos y los problemas propuestos 
son fáciles de comprender y establecer. En ocasiones, se suele atribuir el fracaso en la resolución a la falta de dominio de los procedimientos matemáticos. Sin embargo, se ha verificado con estudiantes de secundaria que las dificultades algebraicas y de cálculo no son el obstáculo principal en la transferencia de aprendizajes en la resolución de problemas. ${ }^{20}$

\section{A MODO DE CONCLUSIÓN}

\section{Propuesta de estrategias didácticas en el aula de química}

A partir de todas las variables anteriormente expuestas que pueden influir en los procesos cognitivos que se realizan para la resolución de problemas, planteamos a continuación un conjunto de estrategias didácticas que podrían aplicarse en el aula de Química:

- Hay que conseguir una comprensión y asimilación conceptual de tema que estamos tratando antes de abordar la resolución de problemas. No se puede pretender conseguir dicha comprensión mediante la resolución de problemas. Una técnica que puede ser utilizada por los profesores para ayudar a los estudiantes en estas tareas es el mapa conceptual.

- Los métodos de enseñanza tradicionales (clases magistrales, resolución de problemas algorítmicos, trabajo individual en exclusiva, etc.) no son compatibles con la consecución del aprendizaje significativo de conceptos ni con el fomento de habilidades cognitivas de alto nivel como la resolución de problemas. Se tienen que desplegar prácticas instruccionales para desarrollar capacidades básicas por un lado (altos niveles de comprensión lectora y de control de la propia comprensión) y de razonamiento científico (explicar, interpretar, razonar críticamente, tomar decisiones, etc.).

- Los profesores han de utilizar metodologías que permitan hacer los conceptos abstractos más accesibles a los estudiantes con baja habilidad en las operaciones formales. Estas metodologías incluirán el uso de materiales concretos, por ejemplo, modelos, fotografías, dibujos, diagramas, etc., con la finalidad de cruzar conceptos abstractos con conceptos concretos.

- El trabajo en grupo en el aula debería ser diseñado para maximizar su función sociocognitiva, de modo que pueda producirse un conflicto beneficioso. La colaboración entre estudiantes tiene que basarse en el intercambio de ideas y opiniones.

- Es necesario promover la comprensión cualitativa de los problemas, antes que ofrecer procedimientos numéricos de resolución. Resulta prioritario sacar a la luz, a partir del enunciado del problema, los conceptos subyacentes, sus relaciones y sus límites, y las teorías y principios de los que forman parte. Las discusiones cualitativas en torno al problema pueden efectuarse mientras los estudiantes resuelven el problema, a petición del profesor, que solicitará en primer lugar razonamientos no matemáticos.

- Proveer a los estudiantes de diversas, continuas y prolongadas experiencias de resolución de problemas. Una vez los estudiantes han derivado y comprendido los procedimientos de resolución en problemas básicos (aplicación de algoritmos), deberían adquirir práctica en resolución de problemas de otro tipo. Por ejemplo, problemas no familiares para el estudiante que requieren aplicación de conocimiento conceptual, capacidad de análisis y de síntesis, llevar a cabo conexiones, tomar decisiones, evaluar los razonamientos, etc. Es también importante practicar estrategias de resolución de problemas familiares a través de múltiples contextos para estimular la generalización.

- Es útil para los profesores saber que se puede cambiar la M-demanda (demanda mental) de un problema sin cambiar su estructura lógica. De este modo, podemos facilitar el éxito de los estudiantes mediante la reducción de la cantidad de información a procesar, esto es, evitando la sobrecarga de la memoria de trabajo.

- Instruir a nuestros alumnos con textos que presenten los nuevos conceptos interrelacionados y organizados, mediante estructuras lingüísticas de baja complejidad léxico-sintáctica, así como facilitar la integración de los nuevos conceptos en sus esquemas previos de conocimiento, favorece la elaboración de modelos mentales para resolver problemas.

- Usar representaciones externas mediante símbolos y objetos para ilustrar la base de conocimiento y su estructura durante la resolución de problemas disminuye la complejidad de las actividades de procesamiento cognitivo. Las representaciones externas pueden ayudar al aprendiz a comprender el enunciado de un problema, explicitar sus condiciones, limitar la carga cognitiva y generar vías de solución para el problema.

- Ofrecer actividades de carácter metacognitivo como parte del currículo científico. Tales actividades deben tratar, entre otros aspectos, la existencia de diferentes tipos de conocimiento (declarativo, procedimental, situacional, etc.), la importancia del conocimiento esquemático de problemas, el uso de heurísticos en la resolución de problemas y la explicación del papel de las estrategias/habilidades metacognitivas en los distintos pasos o procesos de resolución de problemas. Se ha de tener presente el papel decisivo que pueden desempeñar estas estrategias/habilidades en los siguientes pasos de resolución de un problema: planificación, control de progreso, verificación e interpretación de los resultados.

\section{Aplicación de algunas de estas estrategias en la enseñanza de resolución de problemas}

A continuación vamos a dar casos concretos que puedan servir de referencia y de guía al profesor de Química en la planificación de sus estrategias en la enseñanza de resolución de problemas.

Se ha visto que el uso de representaciones (modelos, diagramas, dibujos,...) facilita la tarea a nuestros estudiantes cuando abordan la resolución de un problema. Así, Bodner y Domin ${ }^{21}$ han encontrado que ante el problema: ¿Qué pesa más un litro de aire seco a $25{ }^{\circ} \mathrm{C}$ y 1 atm, o un litro de aire a la misma temperatura y presión saturado con vapor de agua?(Tomad la masa molecular promedio del aire 29,0 g/mol?. La mayoría de estudiantes enfocan su resolución asumiendo como punto de partida que la adición de vapor de agua debe incrementar el peso del aire. Sin embargo, cuando se les presenta una representación similar a la Figura 1 junto al enunciado algunos estudiantes cambian sus razonamientos. Y éstos se dirigen hacia la idea de que la substitución, bien de moléculas de $\mathrm{O}_{2}$ (32 uma), bien de moléculas de $\mathrm{N}_{2}$ (28 uma), por moléculas de $\mathrm{H}_{2} \mathrm{O}$ (18 uma), no hace que la mezcla de gases sea más pesada.

Como ya hemos indicado, es conveniente practicar estrategias de resolución de problemas comenzando por problemas que resulten familiares a los alumnos, esto es, que tengan una gran componente algorítmica, para pasar posteriormente hacia otros problemas menos 

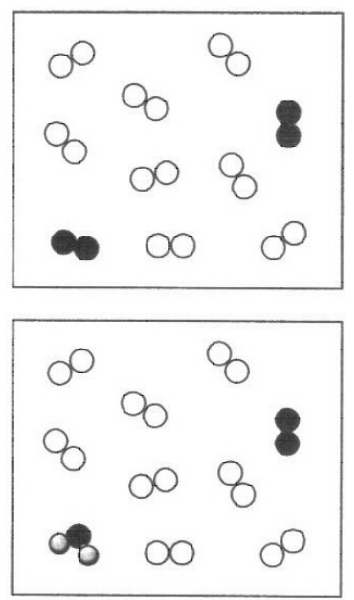

Figura 1. Representación de volúmenes iguales de aire seco y aire saturado con agua. Las moléculas negras son de oxígeno, las blancas de nitrógeno y la restante es agua

familiares, de carácter más abierto y donde al algoritmo desempeña un papel residual. Vamos a presentar a continuación los dos casos extremos de un mismo problema.

Sabiendo que el ácido clorhídrico reacciona con el carbonato de magnesio de acuerdo con la ecuación

$$
\mathrm{MgCO}_{3}+\mathrm{HCl} \rightarrow \mathrm{MgCl}_{2}+\mathrm{H}_{2} \mathrm{O}+\mathrm{CO}_{2}
$$

se pide:

\section{1) Ajustar la reacción química}

2) Determinar la cantidad de substancia ( $\mathrm{mol}$ ) existente en $12 \mathrm{~g}$ de dióxido de carbono.

3) Evaluar la cantidad de substancia de HCl necesaria para producir la cantidad de substancia obtenida en 2).

4) Si partimos de una disolución $2 \mathrm{~mol} / \mathrm{L}$ de ácido clorhídrico, ¿qué volumen de ácido necesitamos para disponer de la cantidad de substancia de 3)

Datos: Masas atómicas relativas de $\mathrm{Mg}, \mathrm{C}, \mathrm{O}, \mathrm{H}$ y Cl: 24,31; 12,$01 ; 16,00 ; 1,01$ y 35,45, respectivamente.

Sabiendo que el dióxido de carbono puede obtenerse añadiendo un ácido a un carbonato, determínese que volumen de ácido clorhídrico de concentración $2 \mathrm{~mol} / \mathrm{L}$ se necesita para producir $12 \mathrm{~g}$ de dióxido de carbono.

En el trabajo de Wood ${ }^{22}$ encontraremos más ejemplos de gradación desde problemas familiares de Química a no familiares. Además, también se analizan los beneficiosos efectos de la discusión en grupos durante la resolución de problemas.

Es muy importante, por necesidades didácticas, saber disminuir la sobrecarga en la memoria de trabajo del estudiante, o lo que viene a ser lo mismo, reducir la demanda mental de un problema. Esto se puede hacer modificando el enunciado u ofreciendo materiales (representaciones, por ejemplo) que faciliten la formación de modelos mentales adecuados del problema. En este sentido, Bodner ${ }^{23}$ nos señala que entre el enunciado del problema: ¿Cuál es la fórmula empírica de un compuesto de cobre y cloro que tiene un porcentaje de cobre del 63,9\% y un 36,1\% de cloro?. Masas atómicas relativas de Cu y $\mathrm{Cl}, 63,55$ y 35,45, respectivamente. Y este otro enunciado: Cuando $9,33 \mathrm{~g}$ de cobre metálico reaccionan con un exceso de gas cloro, se obtienen 14,6 g de un compuesto de cobre y cloro. ¿Cuál es la fór- mula de este compuesto? Masas atómicas relativas de Cu y Cl, 63,55 y 35,45, respectivamente. La diferencia en sus demandas mentales puede llegar a ser decisiva en la resolución exitosa del problema. Así, con este último enunciado del problema, de mayor demanda mental, los estudiantes tienen más dificultades en su resolución.

Recogemos en la Figura 2, porque pensamos que es de gran interés para el profesorado, el heurístico de resolución de problemas propuesto por Lorenzo ${ }^{14}$ en forma resumida y con algunas modificaciones. En él se tienen en cuenta aspectos didácticos básicos en la resolución de problemas y, lo que resulta de mayor relevancia, aspectos metacognitivos que no suelen considerados en las metodologías de enseñanza habitual. Casos prácticos de este heurístico en la resolución de problemas de Química pueden hallarse en el artículo citado de la autora.

1. Leer cuidadosamente el enunciado del problema.

2. Buscar la información que se considere necesaria (en libros de texto, Internet, diccionarios, etc) para entender el enunciado.

3. Identificar qué situación plantea el problema.

4. Identificar qué se busca en el problema y de qué datos disponemos.

5. Determinar cuáles son las magnitudes buscadas y las que son conocidas, expresándolas todas en el mismo sistema de unidades (preferentemente el que recomienda la IUPAC).

6. Organizar la información que proporciona el problema mediante una representación (un dibujo, un diagrama, un gráfico, etc). Esto puede ayudar a comprender mejor la situación que plantea el problema y cómo abordarlo.

7. Construir un "mapa" que muestre las relaciones entre lo que buscamos y los datos de que disponemos, con sus valores y unidades. Si se conocen todas las relaciones, se deben utilizar para resolver el problema. Si no se conocen se debe separar el problema en partes.

8. Separar el problema en partes. En cada parte estableceremos qué magnitudes buscamos y, luego, buscaremos cómo relacionarlas. Haremos uso aquí de principios, leyes, y de cualquier otro fundamento teórico que nos permita encontrar las relaciones buscadas. Puede ser útil en este punto comparar el problema que estamos resolviendo con otros ya resueltos que se piense que muestran algún tipo de similaridad.

9. Una vez se haya descubierto el camino que liga lo que buscamos con los datos disponibles, el problema está cualitativamente resuelto. A continuación, pasaremos a resolverlo cuantitativamente efectuando los oportunos cálculos usando las ecuaciones correspondientes. Hay que ser cuidadoso con los cálculos y las unidades.

10. Examinar si la respuesta al problema es razonable y coherente con el enunciado. Si no es así, hay que pensar dónde podemos habernos equivocado o qué relaciones o conceptos hemos empleado mal, esto es, revisaremos todos los pasos previos.

11. Escribir y razonar qué se ha aprendido resolviendo este problema.

Figura 2. Heurístico de resolución de problemas propuesto por Lorenzo, resumido y con modificaciones

Finalmente, señalar que Hollingworth y McLoughin ${ }^{24}$ proporcionan un completo programa on-line para desarrollar habilidades metacognitivas en la resolución de problemas de Química. Se trata de un programa que se ha aplicado en estudiantes de primer curso universitario, y con él parece que se desarrollan estrategias y procesos reflexivos que inducen a definir, planificar y autocontrolar los razonamientos durante la resolución de problemas.

\section{REFERENCIAS}

1. Costa, S. S. C.; Moreira, M. A.; Caderno Catarinense de Ensino de Física 2001, 18, 263. 
2. Solaz-Portolés, J. J.; Sanjosé, V.; Revista de Educación 2006, 339, 693; http://www.revistaeducacion.mec.es, accessed January 2007.

3. Solaz-Portolés, J. J.; Sanjosé, V.; Educ. Quím. 2006, 17, 372.

4. Solaz-Portolés, J. J.; Sanjosé, V.; Journal of Physics Teachers Education Online, 2007, 4, 25; http://www.phy.ilstu.edu/jpteo, accessed April 2007.

5. Jonassen, D.; Educational Technology: Research and Development 2000, $48,63$.

6. Solaz-Portolés, J. J.; Sanjosé, V.; Ciências \& Cognição, 2008, 13, 192; http://www.cienciasecognicao.org, accessed August 2008.

7. Solaz-Portolés, J. J.; Sanjosé, V.; Revista Mexicana de Psicología 2009, $26,79$.

8. Solaz-Portolés, J. J.; Sanjosé, V.; Asia Pacific Forum on Science Learning and Teaching 2007, 8, article 4; http://www.ied.edu.hk/apfslt, accessed January 2008.

9. Solaz-Portolés, J. J.; Sanjosé, V.; Revista Electrónica de Investigación Educativa 2008, 10, 67; http://redie.uabc.mx/vol10no1/contenidocontenido.html, accessed July 2008.

10. Solaz-Portolés, J. J.; Sanjosé, V.; Revista Electrónica de Enseñanza de las Ciencias 2007, 6, 70; http://saum.uvigo.es/reec, accessed July 2007.

11. Solaz-Portolés, J. J.; Sanjosé, V.; Sísifo. Revista de Ciências da Educação 2008, 6, 105; http://sisifo.fpce.ul.pt, accessed July 2008.

12. Artz, A. F; Armour-Thomas, E.; Cognition and Instruction 1992, 9, 137

13. Taconis, R.; Ferguson-Hessler, M. G. M.; Broekkamp, H.; Journal of Research in Science Teaching 2001, 38, 442.
14. Lorenzo, M.; International Journal of Science and Mathematics Education 2005, 3, 33; http://www.springerlink.com/index/ J61K815288180587.pdf, accessed December 2007.

15. Reed, S. K.; Journal of Experimental Psychology: Learning, Memory, and Cognition 1987, 13, 124.

16. Ross, B.; Journal of Experimental Psychology: Learning, Memory, and Cognition 1987, 13, 629 .

17. Catrambone, R.; Holyoak, K. J.; Journal of Experimental Psychology: Learning, Memory, and Cognition 1989, 15, 1147.

18. Loewenstein, J.; Thompson, L.; Gentner, D.; Psychonomic Bulletin \& Review 1999, 6, 586.

19. Kurtz, K. J.; Loewenstein, J.; Memory \& Cognition 2007, 35, 334.

20. Sanjosé, V.; Valenzuela, T.; Solaz-Portolés, J. J.; Revista Electrónica de Enseñanza de las Ciencias 2007, 6, 538; http://saum.uvigo.es/reec, accessed January 2008.

21. Bodner, G. M.; Domin, D. S.; U. Chem. Edu. 2000, 4, 24.

22. Wood, C.; Chem. Educ. Res. Pract. 2006, 7, 96; http://www.rsc.org/ Publishing/Journals/RP/Index.asp, accessed February 2009.

23. Bodner, G. M. In Toward a Unified Theory of Problem Solving: Views from the Content Domain; Smith, M. U., ed.; Lawrence Erlbaum Associates: Hillsdale, 1991, p. 21-34.

24. Hollingworth, R. W.; McLoughin, C.; Australian Journal of Educational Technology 2001, 17, 50; http://www.ascilite.org.au/ajet/ajet17/hollingworth.html, accessed February 2009. 